\title{
Characterization of noise sources in a rod-airfoil configuration by means of Time-Resolved Tomographic PIV
}

\author{
Lorenzoni V., Violato D. †'and Scarano F. ${ }^{\ddagger}$
}

\begin{abstract}
Time-resolved Tomographic PIV was used to characterize the flow around the leading edge of a NACA 0012 airfoil in rod-airfoil configuration at $R e_{D}=3500$. The volumetric approach at relatively high temporal resolution allows the measurement of the evolution of the 3D vortical structures constituting the Kármán wake of the rod at interaction with the airfoil. The pressure gradient is reconstructed exploiting the definition of Lagrangian derivative and the pressure field is obtained by spatial integration of the Poisson equation. Time-correlation is performed between the aeroacoustic sources of Curle's aeroacoustic analogy and Vortex Sound Theory represented by respectively, time derivative of the pressure fluctuations at the surface and time derivative of the Lamb vector in the surrounding flow. The region of highest correlation is located underneath the airfoil leading edge and appears not to coincide with the region of most intense vortex stretching.
\end{abstract}

\section{Introduction}

YoRTEX-structure interaction noise is of main concern in several aeronautical and industrial applications such as propellers and ventilating systems. The rod-airfoil configuration as benchmark test case for broadband noise predictions of vortex-structure noise has been first proposed by Jacob ${ }^{1}$ in 2004 . Jacob performed planar PIV experiment combined with POD reconstructions to identify the coherent structures in the cylinder wake and compare them with the results of RANS and LES numerical simulations. The analysis lead to the identification of the front part of the airfoil as the main responsible for the noise emission in a rod airfoil configuration. Casalino ${ }^{2}$ developed an aeroacoustic code based on a porous Ffowcs-Williams and Hawkings in combination with URANS simulations for the acoustic prediction. Improvements in the evaluation of the broadband spectral component were achieved by Boudet et al. ${ }^{3,4}$ using LES simulations which allowed the detection of the sub-Kármán vortical structures in the cylinder wake embedded in the larger Kármán rollers which are responsible for emission at higher frequency. In both the cases the characterization of the noise sources relied on numerical data obtained by CFD computations.

The use of experimental data for aeroacoustic investigations of rod-airfoil noise has been only recently proposed. Henning ${ }^{5}$ performed dual PIV and simultaneous far-field microphone measurements on this configuration in order to identify the hydrodynamic features responsible for the acoustic generation by means of correlation-techniques. The results revealed a good capability of the method to individuate the larger structure into the Kármán wake of the rod, although a quantitative estimation of the noise emission was not derived. A deterministic approach based on time-resolved PIV combined with a pressure reconstruction procedure was proposed by Lorenzoni et al. ${ }^{6}$ The pressure evaluated at the airfoil surface constituted the dipolar term of Curle's aeroacoustic analogy which was shown to be responsible for the most of the noise emission in such a configuration. The comparison with simultaneous microphone measurements revealed the ability of the method to reasonably predict the magnitude of the tonal peak of emission and the narrow band spectrum around it. The high frequency component of the predicted spectrum was largely overestimated because of the assumption of in-phase coherent impingement of the vortical structures along the full airfoil span. Further uncertainties were attributed to the lack of 3D flow information for the pressure reconstruction and to insufficient time resolution of the velocity field.

*PHD researcher, Delft University of Technology / Aerospace Engineering Department, Aerodynamics section

$\dagger$ PHD researcher, Delft University of Technology / Aerospace Engineering Department, Aerodynamics section

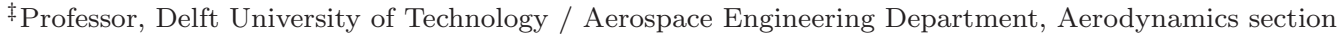


An improved experiment was performed by Violato et al. using tomographic PIV in thin volume configuration at a higher repetition rate around the leading edge of an airfoil in rod-airfoil configuration. The work of Violato showed an improvement in the evaluation of the planar pressure gradient when using a Lagrangian derivative based on $3 \mathrm{D}$ convective acceleration with respect to the purely $2 \mathrm{D}$ case. The effect on the noise prediction was however not yet scrutinized. The present work aims at clarifying the conclusions drawn by Lorenzoni et al. regarding the influence of 3D motion effects on the predicted noise spectra and at the same time, at identifying the aeroacoustic sources in a rod-airfoil flow through volumetric velocimetry measurements.

\section{Theoretical background}

Noise emission in a rod airfoil configuration is mainly due to the interaction of the vortices shed by the rod with the airfoil. ${ }^{1}$ Jacob et al. identified the airfoil leading edge as the region mainly responsible for the noise emission by inspection of the vortex deformation at interaction with the airfoil revealed by instantaneous PIV and hot wire anemometry measurements. The same conclusion was drawn by Lorenzoni et al. ${ }^{6}$ by evaluating the r.m.s of the pressure distribution along the airfoil surface. The latter was justified by the definition of acoustic sources according to Curle's aeroacoustic analogy ${ }^{7}$ in the form

$$
p^{\prime}(\mathbf{x}, t)=-\left.\frac{x_{j}}{4 \pi c_{0}|\vec{x}|} \frac{\partial}{\partial t} \int_{S} \frac{p^{\prime} \delta_{i j}}{r}\right|_{t=t_{e}} n_{i} d y^{2},
$$

in which $p^{\prime}$ on the left hand side represents the propagating acoustic pressure at listener position while $p^{\prime}$ on the right hand side of the equation represents the pressure fluctuation at the body surface. Quadrupolar velocity terms were neglected because of the low Mach number $(<0.02)$ and a free-field Green's function was convoluted with the Lighthill stress tensor. The region of highest pressure fluctuations provides the strongest contribution to the surface integral and the analysis of Lorenzoni et al. showed that the r.m.s. of the pressure fluctuations strongly decays beyond $20 \%$ of the airfoil chord.

Alternative aeroacoustic formulations of vortex-structure interaction noise rely on the use of Green's functions tailored to the geometry of the scattering body (see Gloerfelt et al. ${ }^{8}$ ). In this case the noise predictions can be decoupled from the knowledge of the surface pressure and the radiated pressure can be directly related to kinematic quantities inside the bulk of the fluid (see Powell ${ }^{9}$ and Ffwocks Williams-Hall $^{10}$ for the flat plate). A general formulation including arbitrary compact body geometries was developed by Howe in the Theory of Vortex Sound ${ }^{11}$ by exploitation of the so called Compact Green's Function which takes into account the influence of the body geometry on the radiated field. For far-field listener positions and non vibrating bodies such a formulation reads

$$
p^{\prime}(\mathbf{x}, t)=-\left.\frac{\rho_{0} x_{j}}{4 \pi c_{0}|\vec{x}|^{2}} \frac{\partial}{\partial t} \int_{V}(\vec{\omega} \times \vec{v})\left(\vec{y}, t-\frac{|\vec{x}|}{c_{0}}\right) \cdot \nabla Y_{j}(y)\right|_{t=t_{e}} n_{i} d^{3} y
$$

Here $Y_{j}(y)$ indicates the j-th component of the so called Kirchhoff vector of the body and can be interpreted as the velocity potential of an incompressible flow past the body with unit speed in the $j$ direction at infinity. This encloses the information about the acoustic effect of the body on the sound emission and convoluted with the acoustic sources, indicates the acoustic sources which effectively emit inside the flow.

The acoustic sources in this formulation are represented by volumetric integral of the time variations of the Lamb vector $\vec{\omega} \times \vec{v}$ which can be seen as the time variations of the Coriolis force experienced by the fluid element while moving with the flow. The above formulation offers the great advantage from an experimental point of view of avoiding the evaluation of the surface pressure at the physical boundary.

Due to the limitedness of the observation volume of the present PIV measurement the total acoustic emission of the configuration based on equation (2) could not be estimated. Hence, the present work is limited to the analysis of the correlation of the volumetric kinematic acoustic sources of Vortex Sound Theory $\int_{V} \frac{\partial}{\partial t}(\vec{\omega} \times \vec{v}) \cdot \nabla \vec{Y}_{j}(y)$ with the dynamic sources of Curle's analogy $\int_{S} \frac{\partial p^{\prime}}{\partial t}$. This aims at investigating the equivalence of the two formulations while using experimental velocity data and at spatially identifying the flow regions mostly responsible for the noise emission. 


\section{Experimental apparatus}

Time-resolved tomographic PIV (TR-TOMO-PIV) experiments were carried out in the open test section low-speed wind tunnel $\left(0.4 \times 0.4 \mathrm{~m}^{2}\right)$ of the High-Speed aerodynamics laboratories of TU Delft University. The configuration consists of a plexiglass NACA0012 airfoil of $100 \mathrm{~mm}$ chord length placed at zero incidence $104 \mathrm{~mm}$ downstream of a cylindrical $\operatorname{rod}\left(D_{\text {rod }}=10 \mathrm{~mm}\right)$. Experiments were performed at a free stream velocity of $5 \mathrm{~m} / \mathrm{s}$. An extensive description of the experiment is provided by Violato et al., ${ }^{12}$ the setup is shown in Figure 1.

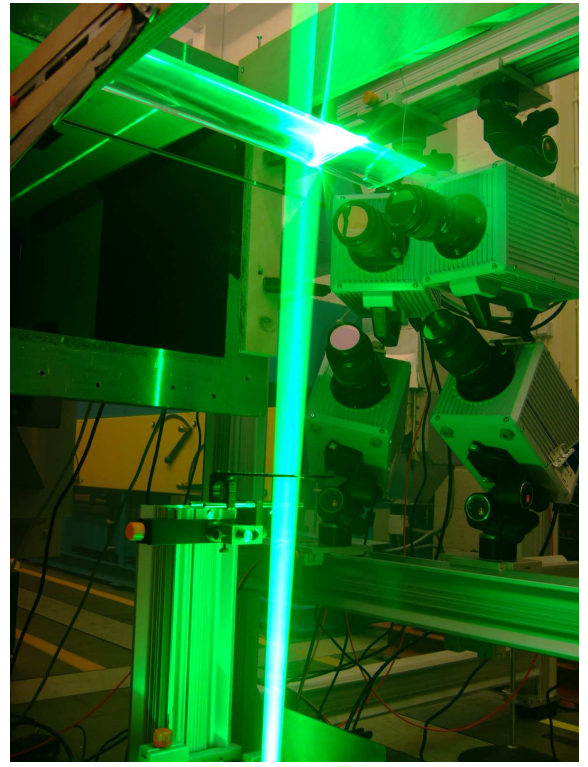

Figure 1. Tomographic PIV setup for the rod-airfoil experiment

The PIV measurement parameters are summarized in Table 1

Table 1. PIV measurement parameters $\left(V_{\infty}=5 \mathrm{~m} / \mathrm{s} D_{\text {rod }}=10 \mathrm{~mm}\right)$

\begin{tabular}{ll} 
Seeding material & Smoke particles $\approx 1 \mu m$ diameter \\
Illumination & Quantronix Nd-YLF \\
& $2 \times 25 \mathrm{~mJ} @ 1000 \mathrm{~Hz}$ \\
Recording device & $4 \times$ Photron Fast CAM SA1 \\
& $\begin{array}{l}\text { CMOS cameras 12-bit } \\
(1024 \times 1024 \text { pixels, } 20 \mu m \text { pixel pitch })\end{array}$ \\
Recording method & double frame/single exposure \\
Recording lens & $f=105 \mathrm{~mm}, f_{\#}=8$ \\
Operational frequency $F s$ & $5000 \mathrm{~Hz}$ continuous mode \\
Illuminated volume & $50 \times 50 \times 3 \mathrm{~mm}^{3}$ \\
Pulse separation $(1 / F s)$ & $0.2 \mathrm{~ms}$ \\
Particles per pixel $(\mathrm{ppp})$ & 0.06 \\
Space resolution & $58.7710^{-6} \mathrm{~mm}^{3} /$ vox \\
Time resolution & $\approx 50 \mathrm{samples} / \mathrm{shedding}$ period \\
Acquisition software & Davis 7.4 \\
\hline
\end{tabular}

At a Strouhal shedding of $100 \mathrm{~Hz}$, the operational frequency of $5 \mathrm{kHz}$ allowed for the acquisition of about 50 velocity fields per shedding cycle. A total ensemble of 5000 images per camera was acquired. The non-dimensional time $t^{*}=\frac{t}{T_{\text {shed }}}$ in the following discussion is normalized with this shedding period $T_{\text {shed }}=1 / 100$. The images acquired by the four high speed cameras were combined together to reconstruct 
the observation volume using the MART algorithm. ${ }^{13}$ The resulting volumes have physical dimensions of $50 \times 50 \times 3 \mathrm{~mm}^{2}$ in respectively the $\mathrm{x}, \mathrm{y}$ and z-axis, discretized with $831 \times 831 \times 50$ voxels (volumetric correspondence of a pixel). The 3D particle displacement was calculated by iterative cross-correlation of pairs of volume subregions ( $47 \times 47 \times 19$ voxel minimum size, $75 \%$ overlap) by means of the VODIM software (Volume Deformation Iterative Multigrid) developed at TU Delft. The resulting 3D velocity field is displaced on a grid of $66 \times 66 \times 6$ points providing a spatial resolution of $0.7 \mathrm{~mm} /$ vector in $\mathrm{x}$ and $\mathrm{y}$ and $0.5 \mathrm{~mm} /$ vector in z direction. The coordinate system is right handed with the $\mathrm{x}$-axis aligned with the free stream velocity, $y$-axis in the direction perpendicular to the chord-span plane and the z-axis in the spanwise direction. The origin of the coordinate system is fixed at the airfoil leading edge at the midspan airfoil plane.

\section{Experimental results}

\section{IV.A. Kinematic quantities}

The tomographic PIV measurement provided the 3D velocity components on a thin volume of 66 planes in $\mathrm{x}$ and $\mathrm{y}$ directions $(0.7 \mathrm{~mm}$ spacing) and 6 planes in the $\mathrm{z}$ direction $(0.5 \mathrm{~mm}$ spacing). This allowed the derivation of all the components of the velocity tensor $\nabla \vec{V}$ and the three components of the vorticity vector $\vec{\omega}$. Consequently also velocity derived quantities as the vortex stretching-tilting vector ${ }^{14}$ and the Lamb vector could be estimated. The data were filtered using a 2 nd order cubic regression of radius 2 in space and 5 in time. This introduces a bias error in the amplitude of $1 \%$ which is comparable with the experimental uncertainty. Velocity derivatives were calculated using a least square regression on a $5 \times 5$ point stencil, which minimizes the measurement statistical error as indicated by Raffel et al., ${ }^{15}$ along the $\mathrm{x}$ and $\mathrm{y}$ direction and a central scheme in the $\mathrm{z}$ direction. Figure 2 provides a direct visualization of the reconstructed

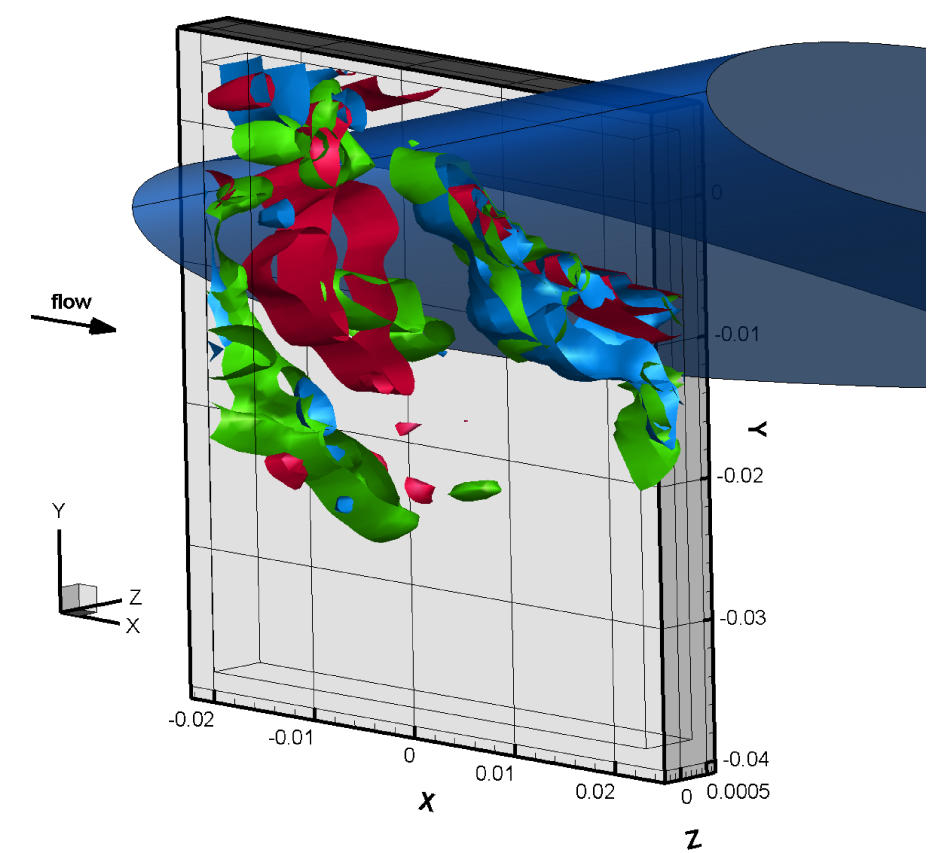

Figure 2. Iso-contours of the vorticity components (level 150 1/s): X-component (blue), Y-component (green), Zcomponent (red)

velocity field with respect to the airfoil position. Isosurfaces of constant vorticity (level $150 \mathrm{1} / \mathrm{s}$ ) are plotted for the three vorticity components. The structures appear mainly $2 \mathrm{D}$ far from the airfoil as indicated by the rather cylindrical shapes which is due to the narrowness of the observation volume with respect to the larger spanwise coherence of the Kármán rollers. The presence of all the three vorticity components reveals non-negligible 3D motion effects approaching the airfoil leading edge. At interaction with the airfoil the spanwise displacement generates a dominating vortical swirling motion around the $\mathrm{x}$ axis.

In high Reynolds number flows the vorticity equation $\frac{D \vec{\omega}}{D t}=\vec{\omega} \cdot \nabla \vec{V}$ indicates that the vorticity of a fluid element along its path is directly related to the three-dimensional term $\vec{\omega} \cdot \nabla \vec{V}$ defined as vortex stretchingtilting vector. The tomographic measurements allowed the evaluation of such a term which is directly related 
to the three-dimensionality of the flow. The magnitude of such vector $(|S T|)$ at the mid-volume z-plane is indicated by the isocontour lines in Figure 3-left. The magnitude of the Lamb vector which is related to the acoustic propagation through equation (2) on the same plane is indicated in Figure 3-right. The latter quantities are superposed to contours of the z-vorticity component for the same snapshot in order to analyze the relative instantaneous local position and spatial extension of the mentioned quantities in comparison with the vorticity field.
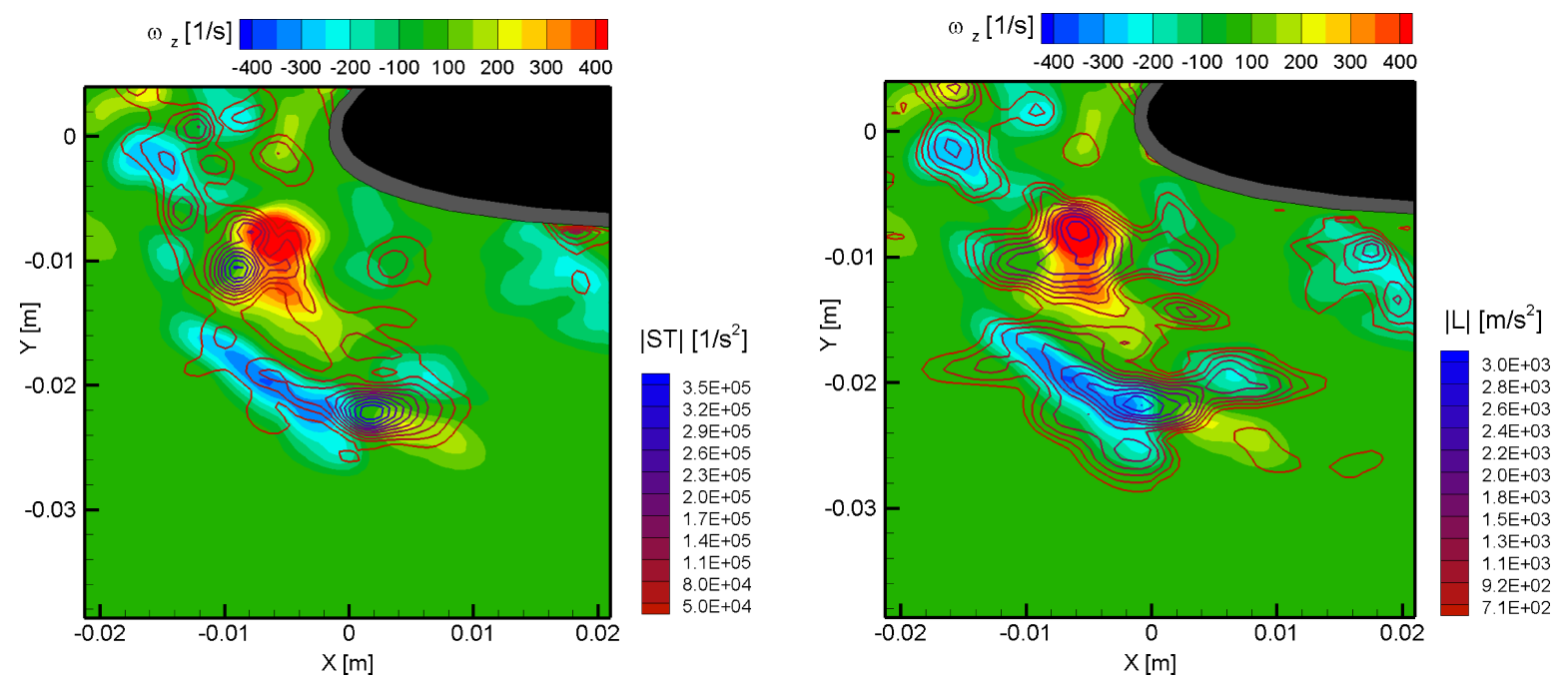

Figure 3. Contours of the instantaneous z-vorticity component at mid-volume z-plane with contour lines of the vortex stretching-tilting vector magnitude (left) and the magnitude of the Lamb vector (right)

From the vorticity contours of Figure 3 it is possible to identify the smaller structures constituting the Kármán wake of the rod. Red blobs indicate anti-clockwise rotating vortices and blue blobs clockwise rotation. The vortex stretching-titling vector features maxima in correspondence of the region between counter-rotating vortices. The Lamb vector magnitude peaks in correspondence of the vortex cores being directly proportional to the vortex acceleration and reveals appreciable levels in the region underneath the airfoil surface.
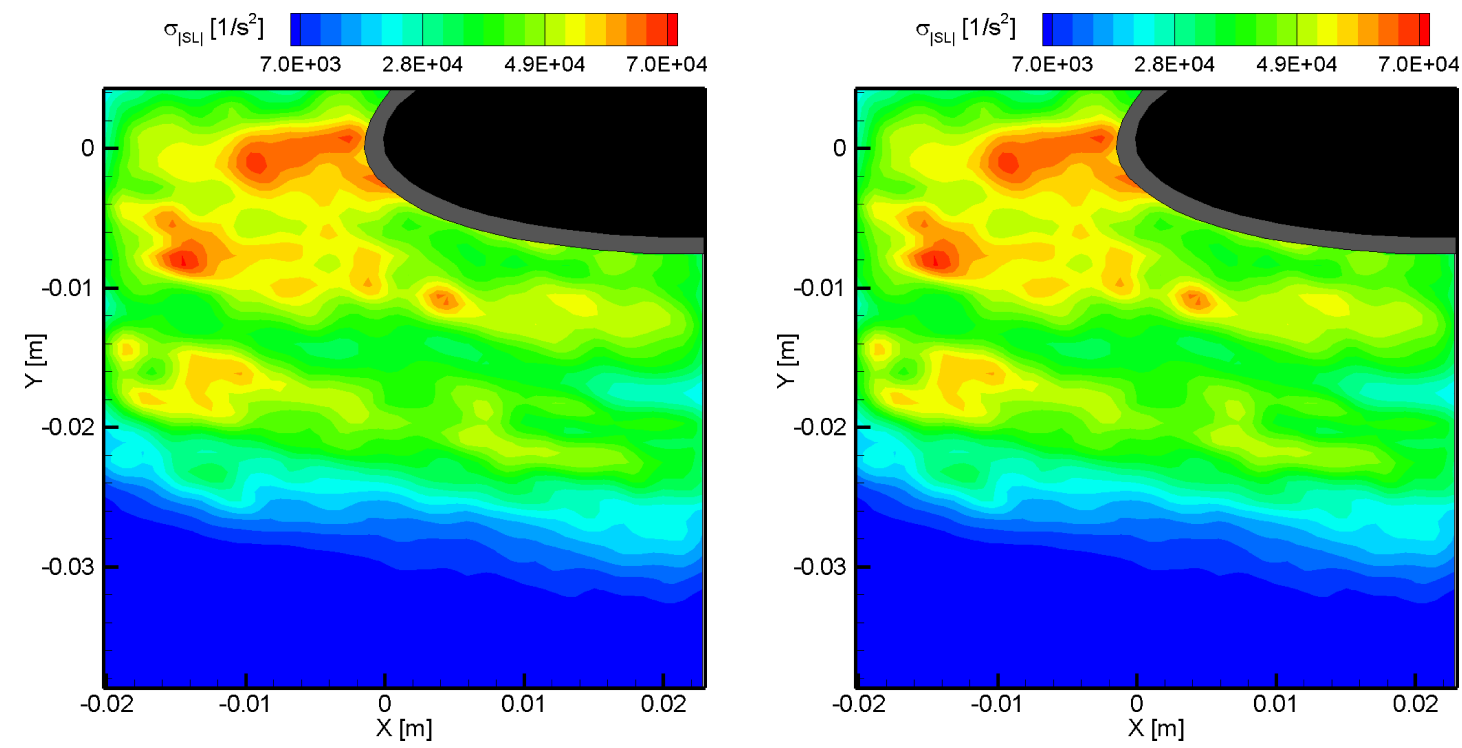

Figure 4. Root mean square of the vortex stretching-titling vector (left) and Lamb vector (right)

The root mean square of the vortex-stretching vector and the Lamb vector allows to visualize the region of strongest temporal fluctuations. Figure 4-left shows that the main vortex stretching is concentrated in the region in front of the airfoil and progressively decreases in the downstream direction. The region of highest 
vortex stretching is localized in a band that extends about $2.5 \mathrm{~cm}$ underneath the airfoil. The strongest fluctuations of the Lamb vector on the other hand are localized at a distance of about $1.5 \mathrm{~cm}$ underneath the airfoil in front of the leading edge and peak at the lower airfoil surface. Negligible magnitude is observed in front of the LE contrarily to the vortex stretching tensor. The Lamb vector exhibits non-negligible fluctuations in a wider band extending down to approximately $3.5 \mathrm{~cm}$ under the airfoil.

\section{IV.B. Pressure deduction}

The reconstruction of the pressure field was carried out by a Planar Pressure Imaging (PPI) algorithm based on the Poisson equation for pressure. ${ }^{16}$ The latter was obtained as divergence of the material acceleration in a Lagrangian approach: ${ }^{17}$ first the particle path between consecutive frames is evaluated assuming a constant convection velocity, then the acceleration is obtained by a first order central difference of the velocities at the estimated positions (see Moore ${ }^{18}$ ). The analysis of the uncertainty on the evaluation of the material acceleration with respect to acquisition time is provided by Violato et al. ${ }^{12}$ The Lagrangian derivatives are calculated using 3D data information. The pressure is therefore calculated by spatial integration of the Poisson equation with boundary conditions of Neumann type at the body surface and Dirichlet in the lower left corner of the domain where fluctuations with the free stream velocity have been shown to be less than $1 \%$. The final pressure field is evaluated on a $2 \mathrm{D}$ plane in the middle of the reconstructed volume. A time interval of $1.2 \mathrm{~ms}$ has been used in the present work for the evaluation of the Lagrangian derivatives, which reduces the precision error on the pressure gradient to less than $10 \%$.

The vortices that constitute the Kármán wake of the rod are convected downstream and interact with the airfoil leading edge generating local pressure fluctuations that partially propagate as sound. In order to emphasize the relation between the kinematic features and the corresponding pressure build up at the surface, the instantaneous pressure field at the midplane of the interrogation volume is drawn together with vectors of the velocity fluctuations in Figure 5 . The velocity fluctuations $\vec{v}_{f l u c t}(x, y, t)=\vec{v}(x, y, t)-\langle\vec{v}(x, y)\rangle$ indicate fluctuations over the local average velocity, where the latter is calculated over 2500 samples corresponding to approximately 50 cycles.
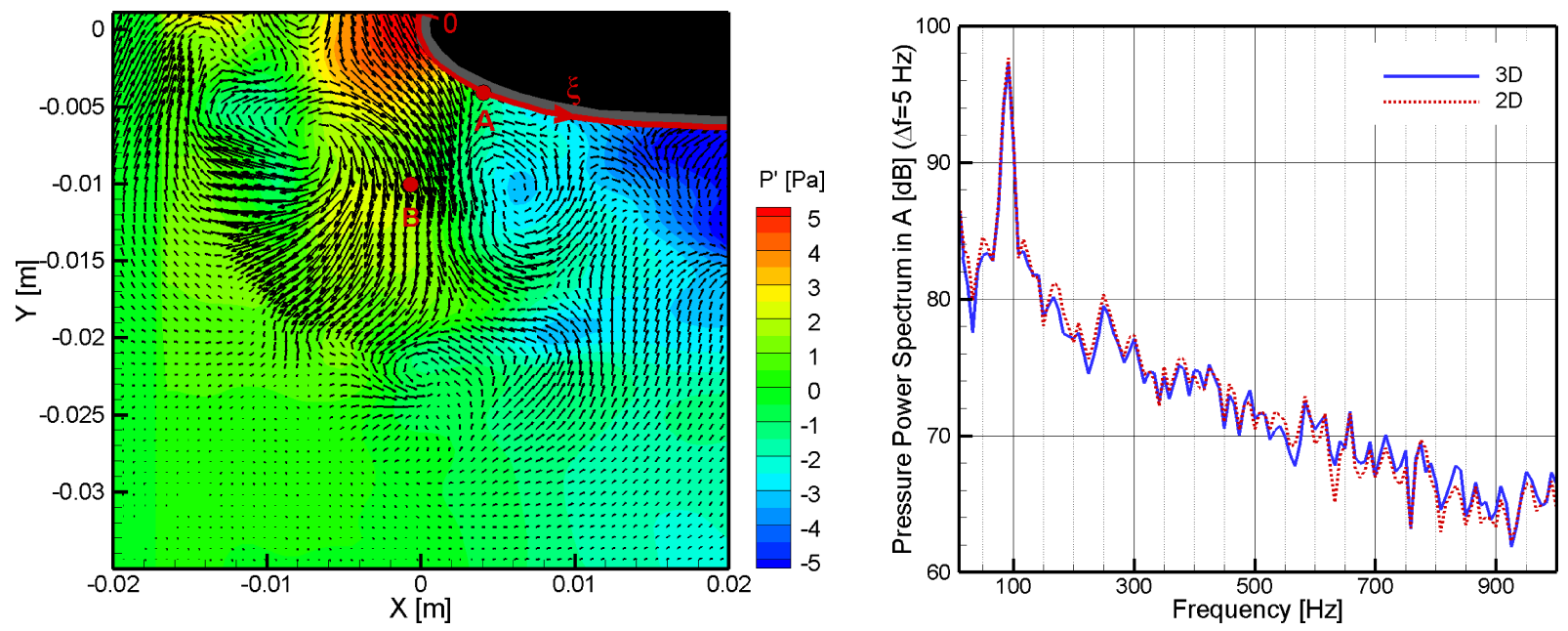

Figure 5. Instantaneous pressure field at the mid-volume z-plane with vectors of velocity fluctuations (left) and power spectrum of the pressure at point $A$ on the surface $\xi$ calculated using $2 D$ and $3 D$ data

Vortices directed towards the airfoil surface produce a local pressure increase while vortices directed outwards induce a local pressure drop. The vortex cores correspond to pressure minima. The grey region in front of the airfoil was masked to avoid unreliable velocity vectors in the pressure reconstruction. The red surface indicated by the coordinate $\xi$ is placed at 3 grid nodes $(1.5 \mathrm{~mm})$ apart from the actual airfoil surface. The points $\mathrm{A}(\xi=0.6 \mathrm{~cm}$ on the surface) and $\mathrm{B}(0,-1 \mathrm{~cm})$ were chosen to check the cross-correlation between surface pressure and Lamb vector (see Section IV.C). The power spectrum of the pressure fluctuations at point A was calculated on 50 cycles (bin $=800$ samples, $50 \%$ overlap) using both $3 \mathrm{D}$ and $2 \mathrm{D}$ (corresponding to the mid-volume z-plane) velocity data for the evaluation of the material derivatives. The spectra feature a strong tonal peak at about $90 \mathrm{~Hz}$ which is slightly lower than the nominal vortex shedding frequency of the rod alone case. The same phenomenon was observed by Casalino et al. ${ }^{19}$ and Lorenzoni et al. ${ }^{6}$ and was 
attributed to an hydrodynamic feedback of the airfoil onto the rod shedding mechanism. The comparison of the spectra reveals minimal changes between the $2 \mathrm{D}$ and the $3 \mathrm{D}$ case similarly to what concluded by Violato $^{12}$ for the pressure time signal at a point in front of the airfoil. Minor discrepancies are randomly distributed along the spectrum with no main concentration on specific frequency bands. The difference of the peak magnitude is negligible for the two cases probably because of the higher signal to noise ratio. This analysis complements the discussion on the spectra obtained by Lorenzoni et al. ${ }^{6}$ and excludes the 3D motion effects from the causes of the over-prediction of the high frequency components of the predicted spectra.

Vortices shed by the rod partially loose coherence while convected towards the airfoil and undergo strong 3 D deformation at interaction with the leading edge (see Boudet ${ }^{4}$ and Lorenzoni ${ }^{6}$ ) the vortex deformation is believed to be directly responsible for the acoustic emission. In order to visualize such link between vortexairfoil interaction and the relative pressure distribution at the airfoil surface a sequence of instantaneous z-vorticity contours are plotted in Figure 6 (top) with the simultaneous pressure distribution at the surface (bottom).
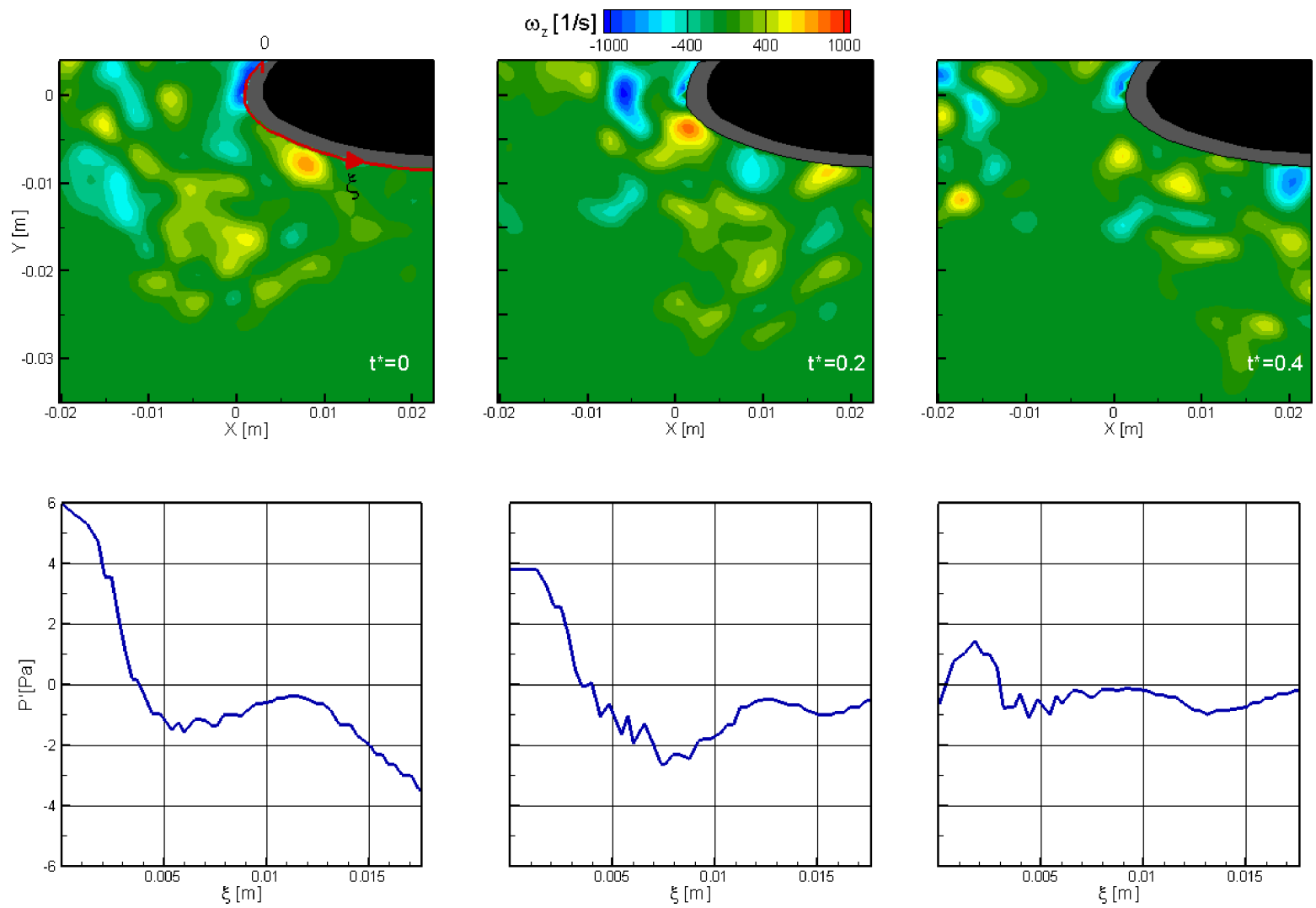

Figure 6. Contours of instantaneous z-vorticity at the mid-volume plane (top) and pressure distribution along the surface $\xi$

The time of the sequence is normalized with the nominal shedding time $(10 \mathrm{~ms})$ and corresponds to a total observation time of about half cycle $\left(0.4 T_{\text {shed }}\right)$. Convection of the vortices along the airfoil sides can be clearly observed on the lower airfoil surface between the first and the second snapshot. The magnitude of the vortex core decreases while convecting downstream. The pressure distribution at the surface features a complex behavior depending on the rotation direction of the vortices. The clockwise rotating vortex (blue) impinging above the airfoil surface above the LE generates an amplitude hump at around $\xi=0$ which gradually decreases as the vortex looses its strength. A second hump in the pressure distribution is observed in correspondence of the anticlockwise rotating (red) vortex underneath the airfoil surface. The position of the hump along $\xi$ moves further as the vortex is convected downstream. The third snapshot $\left(t^{*}=0.4\right)$ does not feature significant vortical levels which explains the relative flatness of the surface pressure distribution. The complex behavior of the pressure distribution is due to the interaction between upward and downward component of each vortex and the relative area of influence. 


\section{IV.C. Aeroacoustic sources identification}

The present work aims at identifying the relation between the aeroacoustic sources represented by the integrals of equation(1) and equation (2). The time-resolved tomographic measurement allowed the evaluation of the time history of the three dimensional quantity $\frac{\partial}{\partial t} \vec{\omega} \times \vec{v}$ inside the bulk of the fluid. The pressure at the airfoil surface was obtained by the PPI method described in Section IV.B. Two-point time correlation was performed between the pressure fluctuations $P^{\prime}$ at point A (in Figure 5) with the fluctuations of the Lamb vector $L^{\prime}$ at point B (P-L). The time signal are plotted together in Figure 7-left and the computed correlation coefficient is shown on the right.
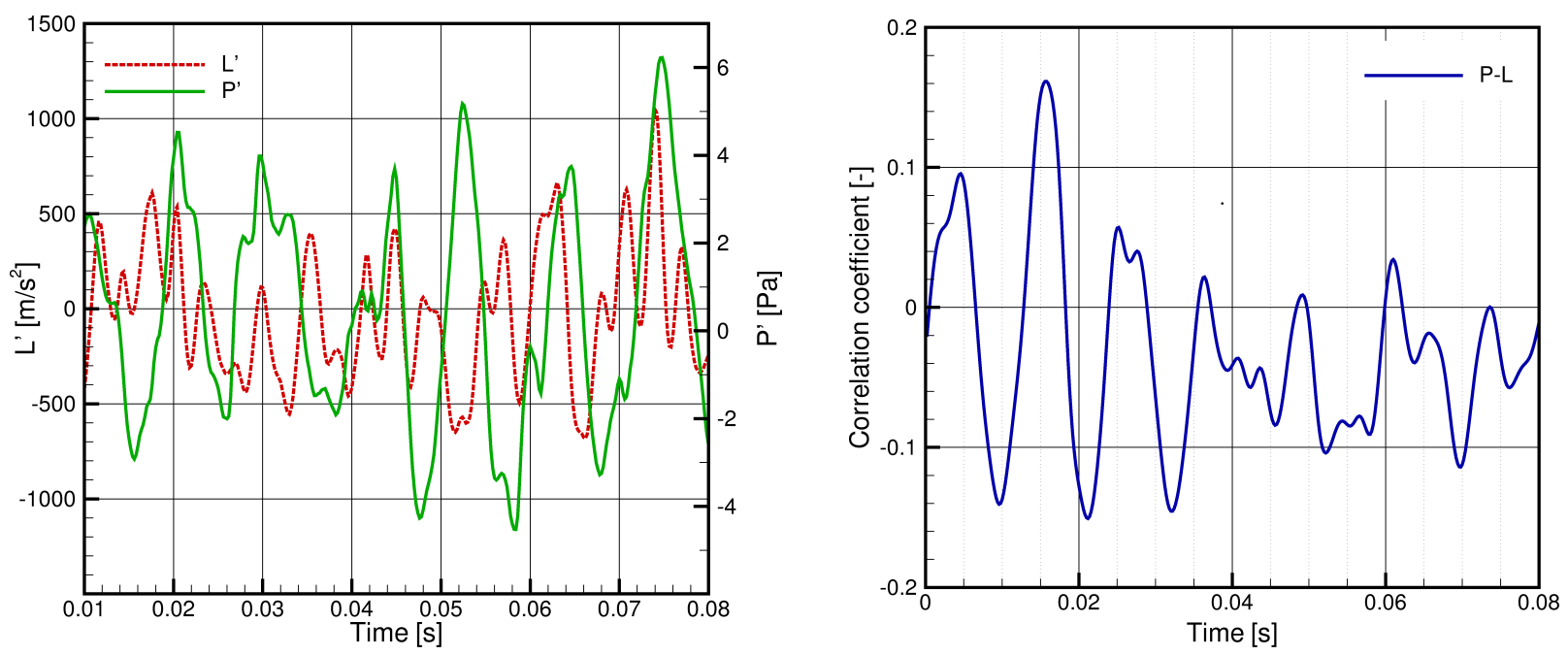

Figure 7. Time history of the pressure fluctuations at point $A$ and Lamb vector fluctuations at point B (left) and corresponding correlation coefficient (right)

The time history of the pressure features a distinguishable periodical pattern every about $10 \mathrm{~ms}$ corresponding to a Strouhal number of $100 \mathrm{~Hz}$. The time signal of the Lamb vector appears to have a more irregular pattern which is due to propagation of experimental uncertainty in the calculation of the velocity gradients in eulerian approach. The fluctuations of the Lamb vector feature a more pronounced low frequency component also confirmed by (non-reported) spectral analysis. The periodicity of the signals is retrieved by the time-correlation on the right. A slight phase shift between the signal can be clearly observed at $\mathrm{t}=$ 0.01 and is confirmed by the correlation coefficient on the right. The delay between the signal corresponds to $5 \mathrm{~ms}$ which indicates a phase opposition of the Lamb fluctuation in B and the pressure fluctuations at $\mathrm{A}$ at the shedding frequency. The correlation coefficient has maximum amplitude of about 1.5 and decays increasing time separation.

In the attempt of visualizing the spatial distribution of the volumetric aeroacoustic sources of equation(2) correlation of the integral quantity $\int \frac{\partial p^{\prime}}{\partial t}$ that represents the source term of Curle's formulation was calculated with the pointwise defined $\left|\frac{\partial}{\partial t}(\vec{\omega} \times \vec{v}) \cdot \overrightarrow{\nabla Y}{ }_{j}\right|$ on the mid-volume z-plane. It is important to notice that the term $\frac{\partial}{\partial t} \vec{\omega} \times \vec{v}$ alone does not directly represent an aeroacoustic source unless convoluted with the specific Green's function tailored to the geometry (in the present formulation the Kirchhoff vector $Y \overrightarrow{(y)}$ specified in equation (2)), which indicates which part of the fluid dynamic sources effectively generates sound. The Kirchhoff vector $\vec{Y}$ in the present case was calculated numerically by use of SchwarzChristoffel conformal mapping ${ }^{20}$ for the NACA0012 approximated with a 2000 point polygonal. The potential flow around the airfoil $Y_{j}$ was mapped into a potential flow around a cylinder of unitarian free-stream velocity in the j-th direction at infinity. The 3D gradients were calculated for each $j$-th component and multiplied in cross-product with the three components of the Lamb vector to determine the acoustic emission in the j-th direction. The $\mathrm{x}$-gradient of the streamwise component of the Kirchhoff vector is shown on the right of Figure 8. 

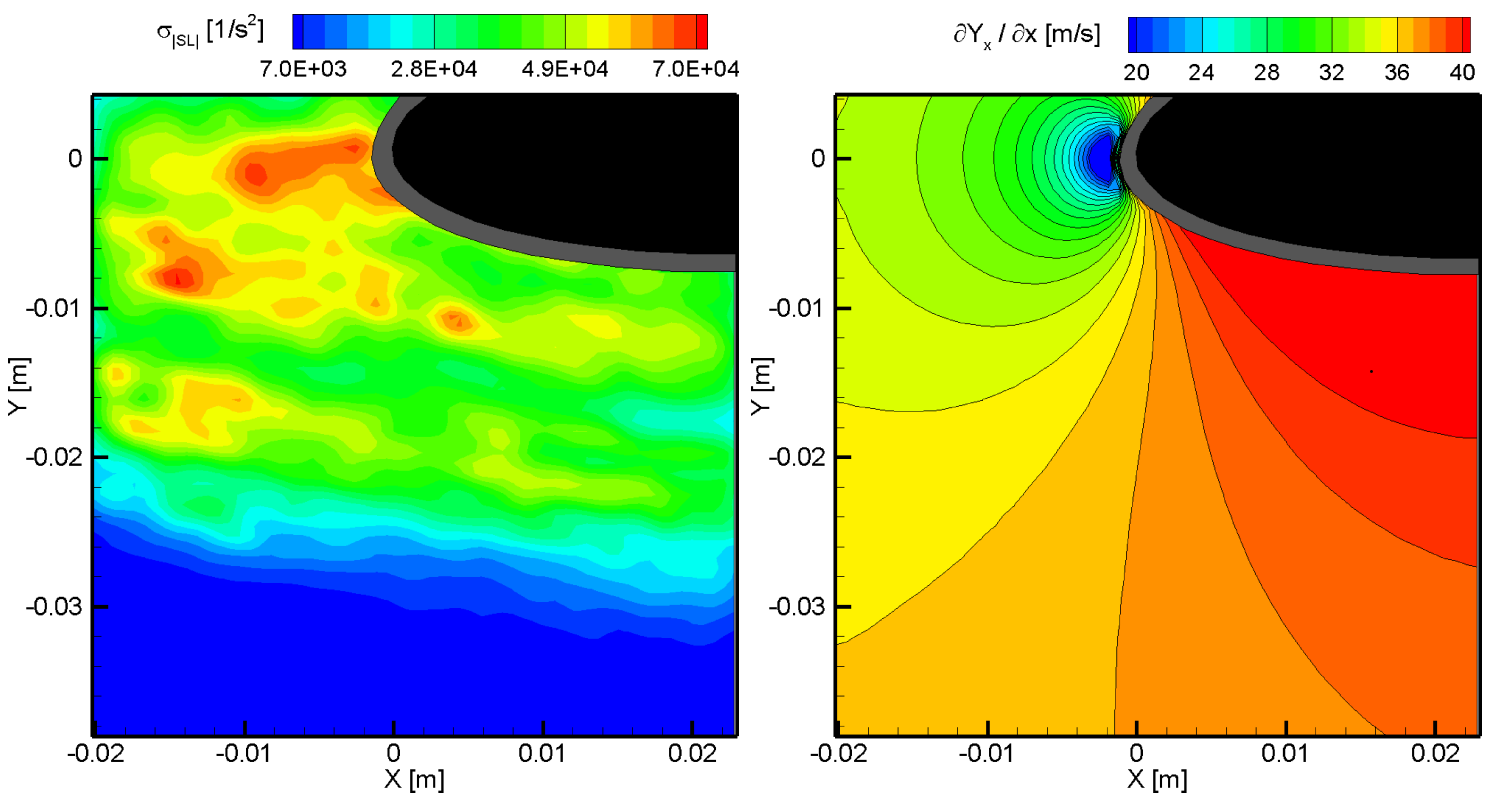

Figure 8. Correlation map of the aeroacoustic sources in equation (1) and equation (2) (left) and streamwise gradient of the x-component of the Kirchhoff vector $Y_{x}$

The map of the correlation coefficients features maxima of approximately 0.27 concentrated in the region underneath the airfoil surface at a distance of about $2 \mathrm{~cm}$ from the surface. Comparison with the r.m.s of the Lamb vector in Figure 4-right shows a similar pattern. The region of strong fluctuations of the Lamb vector at $\mathrm{x}=0.02$ appears to be non correlated with the pressure fluctuations in the part of the surface under consideration. The region in front of the airfoil which was shown in Figure 4-left to feature the strongest vortex deformation appears to be scarcely correlated with the pressure fluctuations at the surface and poorly feasible to be a region of efficient noise generation. The influence of the vortical field in the region under investigation could be felt at distances along the surface which extend beyond the analyzed volume. A complete visualization around the airfoil is needed for a definitive statement. The region of highest correlation is conversely most likely contributing to the acoustic emission.

\section{Conclusions}

A tomographic PIV measurement was performed to reconstruct the 3D velocity field on a thin volume around the leading edge of an airfoil in rod-airfoil configuration. The reconstructed three dimensional velocity field allowed the evaluation of the 3D vorticity vector and derived quantities as vortical stretching-tilting vector and Lamb vector. The vorticity field is dominated by the Kármán vortices shed by the rod and shows a non negligible 3D component which intensifies at interaction with the airfoil. The region of highest vortical distortion on the plane is mainly concentrated in the region immediately in front of the airfoil. Conversely the main fluctuations of the Lamb vector are localized under the airfoil surface and in close proximity of the surface downstream of the LE.

The pressure at the solid surface was derived using a PPI method based on the definition of material acceleration. The power spectra of the pressure at the surface based on $3 \mathrm{D}$ velocity data and $2 \mathrm{D}$ velocity data relative to the $\mathrm{z}$ mid-plane for the evaluation of the Lagrangian derivative have been compared. These present minimal differences distributed along the whole bandwidth, in agreement with the conclusion of Violato et al. and reveal the scarce influence of 3D motion effects to noise level at high frequency of the spectra predicted by Lorenzoni et al. ${ }^{6}$ The impingement of a vortex on the airfoil surface generates a local pressure increase which moves accordingly with the vortex convection. Time correlation of the pressure at the surface with the Lamb vector at a check point in the vicinity of the surface reveals a phase shift of 180 degrees between the signals. The correlation coefficient shows maxima of about 0.16 and is dominated by the Kármán shedding frequency.

A correlation map was calculated between the integral of the aeroacoustic sources of Curle's analogy and Theory of Vortex Sound represented by respectively the time derivative of the pressure fluctuations at the 
surface and the time derivative of the Lamb vector convoluted with the gradient of the Kirchhoff vector. This features maxima in the region underneath the airfoil which is characterized by high fluctuations of the Lamb vector. Conversely the region of highest vortex stretching in front of the airfoil features scarce correlation with the pressure fluctuations at the surface. The limitedness of the observation volume does not enable to draw definitive conclusions about the actual disposition of the aeroacoustic sources. Visualization around the whole airfoil is needed for a more general discussion.

Future works will be devoted at combining tomographic PIV data with numerically computed Green's functions tailored to the body geometry. This would allow by-passing the computation of the pressure at the body surface, which is the region mostly affected by experimental uncertainties.

\section{References}

${ }^{1}$ Jacob, M. C., Boudet, J., Casalino, D., and Michard, M., "A rod-airfoil experiment as benchmark for broadband noise modeling," Theoretical and Computational Fluid Dynamics, Vol. 19, 2004, pp. 171-196.

${ }^{2}$ Casalino, D., Analytical and numerical methods in vortex-body aeroacoustics, Ph.D. thesis, Politecnico di Torino et L'École Centrale de Lyon, 2002.

${ }^{3}$ Boudet, J., Gorsjean, N., and Jacob, M. C., "Wake-airfoil interaction as broadband noise source: a large-eddy simulation," Aeroacoustics, Vol. 4, 2005, pp. 93-115.

${ }^{4}$ Boudet, J., Casalino, D., Jacob, M. C., and Ferrand, P., "Prediction of broadband noise: airfoil in the wake of a rod," 42nd AIAA Aerospace Science Meeting and Exhibit, Nevada, USA, 2004.

${ }^{5}$ Henning, A., Kaepernick, K., Ehrenfried, K., Koop, L., and Dillman, A., "Investigation of aeroacoustic noise generation by simultaneous particle image velocimetry and microphone measurements," Experiments in Fluids, Vol. 45, 2008, pp. 1073-1085.

${ }^{6}$ Lorenzoni, V., Tuinstra, M., Moore, P., and Scarano, F., "Aeroacoustic analysis of a rod-airfoil flow by means of timeresolved PIV," 15 AIAA/CAES Aeroacoustic Conference, Miami, Florida, 2009.

${ }^{7}$ Curle, N., "The influence of solid boundaries upon aerodynamic sound," Proceedings of the Royal Society of London, Vol. A 231, 1955, pp. 505-514.

${ }^{8}$ Gloerfelt, X., Pérot, F., Bailly, C., and Juvé, D., "Flow-induced noise formulated as a diffraction problem for low Mach numbers," Journal of Sound and Vibration, Vol. 287, 2005, pp. 129-151.

${ }^{9}$ Powell, A., "Aerodynamic noise and the plane boundary," Journal of the Acoustic Society of America, Vol. 32, 1960, pp. 982-990.

${ }^{10}$ Ffowcs Williams, J. E. and Hall, L. H., "Aerodynamic sound generation by turbulent flow in the vicinity of a scattering half-plane," Journal of Fluid Mechanics, Vol. 40, 1970, pp. 657-670.

${ }^{11}$ Howe, M. S., Theory of vortex sound, University Press, Cambridge, 2003.

${ }^{12}$ Violato, D., Moore, P., and Scarano, F., "Rod-airfoil flow investigation by time-resolved TOMO PIV for aeroacoustics," 8th International Symposium on Particle Image Velocimetry, Melbourn, Australia, 2009.

${ }^{13}$ Herrmann, G. T. and Lent, A., "Tomographic particle image velocimetry," Computers in biology and Medicine, Vol. 6, 1976, pp. 273-294.

${ }^{14}$ Scarano, F. and Poelma, C., "Three-dimensional vorticity patterns of cylinder wakes," Experiments in Fluids, Vol. 47, 2009, pp. 69-83.

${ }^{15}$ Raffel, M., Willert, C., and Kompenhans, J., Particle image velocimetry: a practical guide, Springner, 2007.

${ }^{16}$ van Oudheusden, B. W., "PIV-based forces and pressure measurements," Recent advances in Particle Image Velocimetry, Vol. LS 2007-09, von Karman Institute, Rhode-St-Genèse, 2009.

${ }^{17} \mathrm{Liu}, \mathrm{X}$. and Katz, J., "Instantaneous pressure and material acceleration measurements using a four-exposure PIV system," Experiments in Fluids, Vol. 41, 2006, pp. 227-240.

${ }^{18}$ Moore, P., Lorenzoni, V., and Scarano, F., "Comparison of two techniques for aeroacoustic determination from rod-airfoil PIV experiment," 8th International Symposium on Particle Image Velocimetry, Melbourn, Australia, 2009.

${ }^{19}$ Casalino, D., Jacob, M., and Roger, M., "Prediction of rod-airfoil interaction noise using the Ffowcs-Williams-Hawkings analogy," AIAA Journal, Vol. 41, No. 2, 2003, pp. 182-191.

${ }^{20}$ Driscoll, T. A. and Trefethen, L. N., SchwarzChristoffel Mapping, Cambridge University Press, 2002. 